\title{
La surdétermination du sens par l'emploi abusif de l'article défini pluriel dans la presse écrite ivoirienne
}

Kouakou Kouman FODJO, Ecole Normale Supérieure (E.N.S.), Abidjan, Côte d'Ivoire

La prise en charge du contexte dans l'analyse de la production du sens demeure une problématique qui fait appel aux notions de mot, énoncé, énonciation, sens... De fait, la notion traditionnelle du mot est l'une de celles qui ont sollicité le plus constamment l'attention des linguistes. Sans entrer dans les détails de débats infructueux pour cette analyse, nous retenons que le mot est une notion qui recouvre des réalités diverses et une certaine pertinence dans l'analyse du discours. En effet, c'est par/avec lui que se construit toute unité linguistique de la plupart des analyses modernes en linguistique et en philosophie du langage : l'énoncé (oral et/ou écrit). Ainsi, le «sens linguistique total» de l'énoncé « résulte du sens lexical des mots individuels auquel vient s'ajouter le sens structurel» (Kerbrat 6). En d'autres termes, chaque mot utilisé dans la production d'un énoncé intervient dans le sens global ainsi produit par l'ensemble. C'est pourquoi, dans le cadre de l'analyse de l'énoncé, tout mot ayant servi à sa production n'est pas à négliger.

A ce titre, le mot prend une importance capitale dans n'importe quelle situation de production d'énoncés, notamment dans la presse écrite où les modalités d'émergence du sens sont parfois complexes du fait du diptyque objectivité / subjectivité et de la complexité des instances émettrice et réceptrice. De fait, dans la presse écrite, «l'exigence d'objectivité apparait comme la première qualité » (Koren 24) mais il s'y déploie subrepticement ou même très souvent explicitement la subjectivité. Par ailleurs, le respect strict « des règles contraignantes de la syntaxe » semble s'arrêter et la liberté structurelle du journaliste «s'accroit substantiellement » (Kerbrat 7). Ainsi, certains emplois du déterminant défini pluriel les, des (de + les), aux (à + les), dans bien de journaux ivoiriens en sont une parfaite illustration. Ces emplois abusifs participent de la construction du sens. Des titres et des articles de journaux relatifs à une thématique variée servent de corpus prétexte à l'analyse du rôle et de la valeur de des emplois en question dans l'économie générale du sens.

Nous postulons qu'alors que l'«émetteur complexe » (22) a la possibilité de choisir, parmi la grande classe des déterminants, la sous-classe des indéfinis pluriels qui cadrent en principe avec la situation ou le contexte d'énonciation, il opte pour les définis précédemment évoqués. C'est que ce choix n'est pas neutre, il apporte une plus-value dans l'économie de la production/construction du sens. Notre étude sur la surdétermination du sens puise ses 
principes méthodologiques et conceptuels dans la linguistique de l'énonciation. Au niveau grammatical, le phénomène relève d'une réflexion sur l'analyse du syntagme nominal.

En termes de subdivision, notre analyse comporte trois volets. Le premier instruit, d'une part, sur des aspects généraux de la détermination et, de l'autre, sur la sous classe des déterminants que représente l'article. Il s'agit de faire un bref rappel des notions de détermination, en général, et, de l'article, de manière spécifique, à travers le passage en revue de ses caractéristiques et de ses règles d'usage. Cette étape préliminaire donnera tout son sens au second aspect de l'analyse qui aborde les usages abusifs de l'article défini pluriel à travers un corpus composé d'énoncés extraits de journaux ivoiriens abordant une thématique variée et d'actualité. Le dernier volet relèvera la valeur de ces emplois.

\section{Généralités}

\section{I.1. La notion de détermination}

Vaste notion, la détermination est le fait des grammairiens rationalistes. Habituellement, cette notion renvoie systématiquement au nom. Dans ce cas, on parle de détermination nominale. Toutefois, dans un sens très large, elle s'applique à la «plupart des éléments ayant pour fonction de modifier ou de compléter un élément quelconque considéré comme une constante » (Arrivé et al 220). Il s'agit notamment d'adverbes de temps (demain), de syntagmes prépositionnels (à pied) mais aussi du verbe (détermination verbale). Verbale, elle porte sur les modes, temps et aspects qui, ici, n'intéressent guère notre propos. Nominale, la détermination est considérée comme un mode de fonctionnement du nom, vu qu'elle précise les limites de l'ensemble des signifiés auxquels le nom ou le groupe nominal (GN) déterminés peuvent être appliqués, ainsi que la quantité d'objets auxquels ce nom ou ce GN sont effectivement appliqués.

Au total, la détermination consiste à apporter des limites au concept exprimé, à l'individualiser, à donner une information sur le nom ou le GN. Elle renseigne l'interlocuteur sur les degrés de généralité et d'identification du nom ou du GN considérés. En d'autres termes, la détermination désigne le mode d'introduction, par un morphème qui le précède nécessairement, d'un nom, dans le discours. Ce morphème, le déterminant, peut être un article défini (les dans les enseignants) ou indéfini (une dans une table), un adjectif possessif (mes dans mes enfants) ou démonstratif (ces dans ces livres) ou indéfini (certains dans certains étudiants) ou encore diverses combinaisons de certains d'entre eux (une de mes dans une de mes cousines). Le nom ainsi déterminé peut l'être plus (j'ai vu le professeur de latin) ou moins (j'ai vu un professeur de latin). Aussi est-il possible de traiter, comme des 
déterminatifs, divers composants du syntagme, notamment des adjectifs qualificatifs, des noms compléments, des propositions à valeur déterminative.

\section{I.2. Le déterminant : l'article}

Le terme de déterminant est réservé à un ensemble de morphèmes ayant pour rôle essentiel de permettre l'introduction du nom dans le discours. En grammaire générative, le déterminant est un constituant obligatoire. Il en existe beaucoup. Les déterminants forment une grande classe composite de mots qui en représentent les sous-classes. Parmi elles figurent les articles. Ces derniers sont souvent considérés comme exprimant le degré minimal de la détermination au sein d'un syntagme nominal réduit à sa plus simple expression (article + nom). Ils se répartissent en trois séries de formes : l'article défini, l'article indéfini et l'article partitif.

\section{I.2.1. De l'article défini}

En grammaire française, l'article défini est une sous-catégorie de déterminant défini. Il se présente sous deux formes: les formes simple et contractée. La forme simple, au singulier, comporte trois articles qui sont le pour le masculin, la pour le féminin et l' (devant un nom commençant par une voyelle ou un $h$ muet) s'utilisant à la fois pour le masculin et pour le féminin. Au pluriel, la forme simple se décline en un seul article, la forme épicène, les. La seconde forme, dite contractée, résulte de la fusion des prépositions à ou de avec les formes simples le et les relevées supra. Ainsi, de + le devient alors $d u$ (ex. : la grandeur du $(d e+l e) c h e f)$ et $d e+$ les devient des (ex. : la pudeur des $(d e+l e s)$ hommes $)$. De même, à + le devient au (ex. : le Directeur s'adresse au $(\grave{a}+l e)$ chef) et à + les devient aux (ex. : la note est adressée aux (à+ les) stagiaires). Les articles contractés $d u$ et au déterminent des noms au masculin singulier uniquement alors que des et aux (les formes au pluriel) actualisent des noms aussi bien féminins que masculins. Comme succinctement présenté, l'article défini participe à l'actualisation du nom noyau. Ainsi, il indique que ce à quoi l'on se réfère (la chose, l'animal, la personne) est connu des actants de l'énonciation ou que des satellites vont suivre pour l'expliciter. Ces satellites guideront davantage l'interlocuteur dans la détermination du sens et lui permettront d'identifier précisément le représenté. Comme tel, l'article défini se présente comme l'outil type de la détermination complète. Il désigne surtout un individu déjà identifié et connu par l'énonciateur. En d'autres termes, comme le notent les auteurs de La grammaire d'aujourd'hui : guide alphabétique de linguistique française :

L'article est dit défini lorsqu'il détermine un nom de manière à signaler qu'il désigne une (au singulier) ou des (au pluriel) entité(s) parfaitement circonscrites et identifiables. Cette identification a pour repère un univers de discours représentable par un ensemble d'objets ou d'individus divers, parmi lesquels l'article défini sélectionne un sous- 
ensemble délimité (il se ramène au singleton dans le cas du singulier). Cet ensemble est normalement fourni par la situation ou le contexte. (Arrivé et al 73)

$\mathrm{Au}$ singulier, l'article défini peut désigner une espèce ou un concept dans son ensemble (le lion est le roi des animaux, le travail libère l'homme). Ici, l'on ne fait pas allusion à un lion et à un homme particuliers, mais à l'espèce carnivore (tous les lions) ainsi qu'à l'espèce humaine (tous les humains). Au pluriel, il indique, en principe, que le substantif désigne l'ensemble des membres d'une classe ou d'une espèce (les hommes réfléchissent).

Avec l'article défini, la détermination se présente sous plusieurs formes. En effet, il s'emploie lorsque l'être, l'objet, etc. ont été préalablement mentionnés explicitement dans le même environnement (Il a acheté une (des) pomme(s). La (les) pomme(s) est (sont) succulente(s)).

Par ailleurs, il s'utilise aussi quand le nom est déterminé dans la phrase par un complément (le bureau du chef), une proposition relative (la fille dont j'ai parlé), un superlatif (la plus grave crise ivoirienne).

De plus, l'usage de l'article défini ressortit au sens commun (la lune, la mer), à l'habitude (le Président, la Première dame) ou à la communauté (le dimanche est un jour de repos) et permet d'identifier l'être/l'objet mentionné/spécifique, car étant unique ou faisant partie de l'expérience commune.

L'article défini s'utilise encore dans de nombreuses situations, dont nous retenons ici quelques cas. Ainsi, il apparait devant les parties du corps déterminées par l'emploi de la personne dans la même phrase (il se brosse les dents après le repas, il a très mal à l'estomac), devant les jours de la semaine et les dates (le lundi, le vendredi, les 8et 9 Septembre 2012), les noms géographiques et le nom de certaines villes (le Japon, la Côte d'Ivoire, le Cap, la Haye), devant un terme, une langue ou une discipline connus de tous et concernés dans leur ensemble (la corruption, la mondialisation, le français, la grammaire), une catégorie tout entière (il étudie les serpents).

\section{I.2.2. De l'article indéfini}

L'article indéfini se présente sous trois formes dont deux au singulier à savoir un (masculin) et une (féminin) et une seule, des, pour le pluriel. Tout comme l'article défini, l'article indéfini est une sous-catégorie du déterminant. Toutefois, il est indéterminé ou partiellement déterminé. Il s'emploie devant des êtres ou des choses que l'on peut compter mais qui ne sont pas encore identifiés et connus par l'énonciateur. Les causes de l'indétermination de l'article indéfini sont nombreuses. En voici quelques-unes. 
Lorsque l'identité individuelle d'un être, d'une chose, d'un objet n'est pas spécifiée, c'est-à-dire qu'on peut le/la désigner par n'importe lequel/laquelle (un professeur de grammaire), alors s'utilise l'article indéfini. Aussi, quand un représentant typique d'une catégorie (un élève consciencieux étudie) ou une seule variété du nom dans un groupe plus grand est considérée (une sorte de..., une espèce de...), l'article indéfini est de mise.

\section{I.2.3. De l'article partitif}

C'est le troisième et dernier type des articles. Il est formé à partir de de + article défini, mais au masculin et au pluriel, on opère une contraction qui donne $d u$ et des. Il se neutralise en $d e$ ou $d^{\prime}$, à la forme négative. L'article partitif s'emploie pour introduire une partie ou une quantité d'une substance que l'on ne compte pas (de l'air, du retard, etc.).

L'emploi de l'article partitif est lié au sens du verbe de la phrase comme le prouvent les exemples suivants : (Il achète du café) mais (Il adore le café). L'article partitif est répété devant les noms mis en série (ma fille veut du chocolat, du pain, de la confiture et des oufs).

En définitive, les articles du français indiquent des degrés de la détermination du nom ou du syntagme auquel ils sont associés en apportant avec eux une indication visible du genre et du nombre du nom ou du syntagme qui les suit. Cette indication est une contrainte grammaticale imposée par le nom puisque les articles s'accordent, comme les adjectifs, avec le nom qui les accompagne. De la sorte, l'usage de l'article défini laisse comprendre que le nom ou le syntagme employé est correctement identifié par l'énonciateur. Il est donc possible d'expliquer les raisons de cette identification et de les justifier en observant la façon dont le nom est déterminé dans une phrase concernée. Autrement dit, le contexte linguistique héberge l'ensemble. Quant à l'usage de l'article indéfini, il obéit à ce qui suit. L'objet désigné par le nom employé n'a pas encore été identifié dans les circonstances d'énonciation. On sait seulement qu'il s'agit d'un/des objet(s) particulier(s), d'un cas intéressant, mais la phrase ne donne pas encore les informations qui permettent de les/l'individualiser. Par l'usage de l'article partitif, on comprend que l'objet désigné par le nom employé peut être mesuré en termes de quantité (un peu de, beaucoup de). On sait par ailleurs que cette portion de n'est pas explicitement présentée dans la phrase concernée.

En dépit de tout cet arsenal grammatical, pour utiliser sans ambiguïtés l'article défini pluriel, on observe, dans la presse écrite ivoirienne, une confusion dans son usage.

\section{Les emplois abusifs du défini pluriel dans la presse écrite}

L'article défini pluriel se présente sous trois formes comme nous l'avons précédemment relevé. Chacune de ces formes fait l'objet d'usage qui ne coïncide pas toujours 
avec les usages prescrits et reconnus. Pour analyser ce fait, nous prenons pour prétexte un corpus extrait de neuf quotidiens, choisis sans critère préalable. Toutefois, on y trouve des journaux de toutes les sensibilités politiques (voir le détail dans le corpus), puisque, la presse écrite ivoirienne, ce n'est un secret pour personne, dans son ensemble, est idéologiquement typée. Ce corpus porte sur une thématique variée et les énoncés à analyser sont majoritairement des titres d'articles de journaux auxquels s'ajoutent des extraits de l'exploitation de certains titres.

\section{II.1. Présentation détaillée et explication du corpus}

L'article défini accompagne un nom, un syntagme, un groupe nominal prépositionnel, etc. qu'il détermine. La présentation du corpus se fera par rubriques, en fonction des éléments déterminés par l'article. Cette présentation facilitera l'exploitation que nous en ferons. Ainsi il se présente comme il suit :

\section{II.1.1. Article défini les + nom.}

1. Les Ivoiriens jugent le gouvernement. Nord sud 2406 du 10/08/13, p.1.

2. «Ce n'est pas dans les mosquées seulement qu'on a tué en Côte d'Ivoire. On a tué dans les églises, on a tué dans des buissons... ». Le nouveau courrier 732 du 21/02/13, p.3.

3. Au total, ce débat arrive dans un contexte sociopolitique vicié qui ne favorise pas l'acceptation aisée et sincère par les populations. Notre voie 4472 du 19/07/13, p.2.

4. Toujours à Bonon, à ces mêmes législatives, les militants du RDR, voulant intercepter les urnes, ont attaqué à l'arme un convoi de l'Onuci. Notre voie 4472 du 19/07/13, p.5.

5. Voici les secrets de l'opération moustique et maracana de nuit. L'expression $1164 \mathrm{du}$ 29-30/06/13, p.1.

6. Mais bien décidée à y mettre fin, la tutelle veille au grain et les indélicats sont tombés dans les filets des agents du ministère. L'expression 1164 du 29-30/06/13, p.6.

7. Education nationale, les instituteurs assiègent la Fonction publique. Notre voie 4472 du 19/07/13, p.1.

8. Bloléquin, les FRCI attaquent des paysans Baoulé. Notre Voie 4472 du 19/7/13, p.1.

9. Déchets toxiques, les victimes manifestent devant la Présidence. Notre Voie $4472 \mathrm{du}$ 19/07/13, p.9.

En 1, ceux qui donnent leur avis sur le gouvernement, interrogés au hasard, dans le cadre d'un micro trottoir, peuvent ne pas être tous Ivoiriens. De plus, s'ils le sont tous, admettons-le, ils ne sont pas représentatifs de la majorité des Ivoiriens, encore moins de la totalité. C'est pourquoi, il ne saurait être question de les Ivoiriens, mais d'Ivoiriens. Ainsi, 1 devient «des Ivoiriens jugent le gouvernement». De même, en 2, pour avoir été témoin de 
l'histoire récente de la Côte d'Ivoire, nous savons que plusieurs endroits, dont des mosquées aussi bien que des églises, ont été le théâtre de violences meurtrières. Toutefois, il ne faut pas généraliser, mais nuancer comme l'auteur l'a fait pour buissons dans lesquels des meurtres ont été aussi commis. Ici, on devra plutôt avoir «des mosquées, des églises ». Le même raisonnement infirme le choix du déterminant défini les au profit de déterminants indéfinis, car il ne s'agit pas de : les populations, les urnes, les secrets, les indélicats, les instituteurs, les FRCI, les urnes, les victimes mais bien de partie de celle-ci ou de ceux-ci dans les autres énoncés de 3 à 9.

\section{II.1.2. Article défini les + syntagme nominal}

10. Enfin : les soutiens de KKB se dévoilent. Le jour 2798 du 01/08/13, p.1.

11. Les $S G$ de section apportent leur soutien à Bédié. Le mandat 1154 du 01/8/13, p.1.

12. Les barons du PDCI en colère contre Bédié. Notre voie 4464 du 10/07/13, p.1.

13. Les chefs Bété demandent pardon à Ouattara. Le patriote 4108 du 01/08/13, p.1.

14. Les chefs d'Etats africains annoncent la rupture avec la CPI. Le quotidien d'Abidjan 0914 du 28/05/13, p.1.

15. Arrestation des coupeurs de route, les ex-combattants pro-Ouattara revendiquent les attaques répétées. Le quotidien d'Abidjan 0914 du 19/07/13.p.1.

16. Toujours à Bonon, à ces législatives, les militants $d u R D R$, voulant intercepter les urnes, ont attaqué à l'arme un convoi de l'ONUCI. Notre voie 4472 du 19/07/13, p.5.

17. Déstabilisation du régime Ouattara : les pro-Gbagbo ne finiront pas de surprendre les Ivoiriens sur leur entêtement [...]. L'expression 1164 du 29-30/06/13, p.2.

18. Cependant, elles dénoncent une autre sorte de fraude où «les candidats libres sont transformés en candidats officiels ». L'expression 1164 du 29-30/06/13, p.6.

19. L'ONU aux trousses des criminels pro-Ouattara. Notre voie 4483 du 01/8/13, p.1.

20. Les enseignants du primaire attendent toujours leur salaire. Notre voie $4472 \mathrm{du}$ 19/07/13, p.9.

21. Résultats scolaires : les parents d'élèves mécontents. Notre voie 4472 du 19/07/13, p.9.

22. La spécificité de cette parade militaire a été, une fois de plus, la surprise de voir les armées africaines participer à ce défilé. Le quotidien d'Abidjan 0914 du 19/07/13, p.2.

Les énoncés en sus suscitent les interprétations et les commentaires suivants.

En 10, il ne fait l'ombre d'aucun doute que le leader de la jeunesse du PDCI (Parti politique ivoirien) a des soutiens (réels et/ou virtuels). Toutefois, presque tous sont encore tapis dans l'ombre pour l'instant. Bien qu'il soit clair qu'ils se dévoileront, ils ne le feront certainement pas concomitamment, mais chacun à son rythme. C'est donc un processus. Or 
en l'état, tel que c'est écrit et donc dit, nulle part, le processus n'est évoqué. Qui plus est, le verbe se dévoiler signifie apparaitre, se manifester ouvertement. Sur cette base, si nous procédons à une commutation, l'énoncé deviendra: les soutiens de KKB se manifestent ouvertement (entendu, tous). Or, ce n'est pas le cas, car d'autres suivront certainement. Pour être plus objectif, il aurait été mieux de donner, ou le nombre exact de ceux qui se sont dévoilés ou, à défaut, d'utiliser l'un des indéfinis, notamment certains, bon nombre, quelques..., ou encore relever que ces derniers commencent $\grave{a} . .$.

Pareillement, Bédié, en 11, ne peut avoir ou, pour être plus précis, n'a pas le soutien des (entendu de + les, c'est-à-dire la totalité) SG de section, vu que certains sont favorables au candidat déclaré, KKB, son futur adversaire. Aussi est-il illusoire que les barons du PDCI, c'est-à-dire tous, en 12, se liguent contre Bédié, surtout que lui-même et nombre de ses partisans sont eux aussi des barons du parti. En outre, il n'est pas certain que tous les chefs Bété, en 13, sauf par crainte ou peur de représailles, par hypocrisie ou par mimétisme, demandent pardon.

Il en est de même pour les chefs d'Etats africains, les ex-combattants pro-Ouattara, les militants du RDR, les pro-Gbagbo, les candidats libres, des criminels pro-Ouattara, les enseignants du primaire, les parents d'élèves, les armées africaines, dans les énoncés 14 à 22. Envisager la détermination comme totale avec les référents actuels, c'est surenchérir leur sens. Il ne peut s'agir que d'une présomption de la totalité ou de détermination totale.

\section{II.1.3. Autres déterminants définis : des $($ de $+l e s)+n o m$, des $($ de $+l e s)+G N$ aux $(\grave{a}+l e s)+$ nom et tous / toutes + les + GN}

23. Violence politique, coup d'Etat, rébellion armée RDR : les mains souillées du sang des Ivoiriens. Notre voie $4472 \mathrm{du}$ 19/07/13, p.1

24. Les massacres des autochtones ne sont-ils pas la marque de leur volonté de conquête de l'espace ivoirien? Notre voie 4472 du 19/07/13, p.2.

25. A la grande surprise des Ivoiriens, Houphouët et son $1^{\mathrm{er}}$ ministre refusent de sanctionner les militaires qui se sont rendus coupables des tueries. Notre voie $4472 \mathrm{du}$ 19/07/13, p.4.

26. L'ONU aux trousses des criminels pro-Ouattara. Notre voie $4483 \mathrm{du}$ 01/8/13, p.1.

27. Ce qui est ainsi décrit est bien la réalité du drame ivoirien, tel que Gbagbo et les siens l'ont infligé à la Côte d'Ivoire et aux Ivoiriens. Le patriote 3975 du 21/2/13, p.2.

28. Ils doivent également se poser [...] les vraies questions. Si avec toutes les forces de Côte d'Ivoire en notre disposition, nous n'avons pas pu vaincre Alassane Ouattara, est-ce maintenant que nous y parviendrons ? L'expression 1164 du 29-30/06/13, p.4. 
29. Tous les cadres du FPI et des partisans de Laurent Gbagbo ont vu leurs biens emportés par la violence du RDR. Notre voie 4472 du 19/07/13, p.5.

L'analyse similaire à celle des énoncés précédents conduit aux mêmes conclusions. Le déterminant défini, en réalité, ne désigne que des référents parfaitement indéfinis ou partiellement déterminés. Ainsi, qu'il s'agisse des Ivoiriens, des autochtones, des criminels pro-Ouattara, ou que ce soit aux Ivoiriens, toutes les forces de Côte d'Ivoire, tous les cadres du FPI, l'usage du déterminant défini est hyperbolique.

Le constat est, en définitive, le même. L'usage du déterminant défini ne correspond pas toujours à ses emplois normatifs. Le sens des énoncés construits sur cette base s'en trouve, du point de vue logique, fortement modifié. Pour preuve, procédons à l'analyse syntaxique de leurs structures.

Considérons les noms déterminés par les différents articles définis comme des constantes. Désignons par X, le nom; par Y, le nom ou le GN qui aident à actualiser X. La structure syntaxique des expressions à analyser se présente sous les formes suivantes :

Les + X. Ex. : les Ivoiriens $(1)=$ les $+\mathrm{X}(\mathrm{X}$ équivaut à Ivoiriens) et ainsi de suite.

Les $+\mathrm{X}+\mathrm{Y}$. Ex.1: les chefs Bété $(13)=\mathrm{Les}+\mathrm{X}+\mathrm{Y}(\mathrm{X}=$ les chefs, $\mathrm{Y}=$ Bété $)$;

Ex.2: les $S G$ de section $(12)=$ les $+\mathrm{X}+\mathrm{Y}(\mathrm{X}=S G, \mathrm{Y}=$ de section $)$.

Des $(\mathrm{de}+\mathrm{les})+\mathrm{X}$. Ex.: des Ivoiriens $(25)=\mathrm{des}+\mathrm{X}(\mathrm{X}=$ Ivoiriens $)$.

Des $(\mathrm{de}+\mathrm{les})+\mathrm{X}+\mathrm{Y}$. Ex.: des criminels pro-Ouattara $(26)=\mathrm{des}+\mathrm{X}+\mathrm{Y}(\mathrm{X}=$ criminels, $\mathrm{Y}=$ pro-Ouattara).

Aux $(\grave{a}+$ les $)+$ X. Ex. : aux Ivoiriens $(27)=$ aux $+X(X=$ Ivoiriens $)$.

Toutes les/ tous les + X+Y. Ex. : toutes les forces de Côte d'Ivoire (28) (X = forces, $\mathrm{Y}=$ de Côte d'Ivoire).

Ex. : tous les cadres $d u$ FPI (29), tous les $+\mathrm{X}+\mathrm{Y}(\mathrm{X}=$ cadres, $\mathrm{Y}=d u$ FPI).

On peut les réduire et les réécrire plus simplement :

- déterminant défini $+X$,

- déterminant défini $+\mathrm{X}+\mathrm{Y}$

Du point de vue syntaxique, la configuration du corpus est celle qui vient d'être présentée. Les déterminants, dans ces structures, actualisent des référents $\mathbf{X}$, d'une part et, $\mathbf{X}+\mathbf{Y}$, de l'autre. La première structure, déterminant $+X$, ne contient pas d'expansion, cependant que dans la seconde, déterminant $+X+Y, \mathrm{Y}$ constitue l'expansion qui aide à l'actualisation de X. Ces divers éléments syntaxiques ou satellites affinent l'actualisation de $\mathrm{X}$ et permettent mieux, en principe, d'identifier les différents référents. 
Ces structures nous inspirent quelques réflexions. A priori, il ne semble se poser aucun problème de compréhension de ces énoncés. Autrement dit, le récepteur perçoit l'information à lui transmise par/via le journaliste. Toutefois, à l'analyse, si sur le nombre pluriel le consensus se fait, le problème se pose quant à la détermination. Les syntagmes, dans les énoncés, sont-ils réellement déterminés totalement, ainsi que l'autorise le sens qui découle de l'usage des déterminants définis pluriels?

Les aspects linguistiques mais aussi logiques, facteurs importants dans la détermination, indiquent que les référents dans nos énoncés sont en réalité partiellement déterminés. En effet, les structures se traduisent comme suit :

- déterminant défini $+\mathrm{X}=$ tous les $\mathrm{X}$ ou la totalité des $\mathrm{X}$,

- déterminant défini $+\mathrm{X}+\mathrm{Y}=$ tous les $\mathrm{X}+\mathrm{Y}$.

Or à la lecture des énoncés, il ne s'agit pas de tous les $X$ ou de tous les $X+Y$. Sur cette base, on peut faire les interprétations suivantes.

Sur le plan logique, l'usage du déterminant les ou de ses formes contractées suppose que le référent est un groupe, une famille, un type, etc. indissociables, entiers. En d'autres termes, l'article défini pluriel exprime la totalité du référent. A notre avis, il y a donc manifestement surdétermination dans les énoncés du corpus. Car, même si $\mathrm{X}$ ou $\mathrm{X}+\mathrm{Y}$ renvoient à des référents, ces référents ne sont pas entiers comme l'explication des énoncés l'a montré. Seuls quelques membres, une partie ou une portion sont concernés par la détermination.

Par ailleurs, l'objectivité si chère à la presse, exige une désignation objective des référents pour un sens global objectif.

Si les déterminants définis ne coïncident pas avec la situation, le contexte ou l'environnement des énoncés du corpus, en revanche, les déterminants indéfinis prospèreront dans un tel environnement pour déterminer les $\mathrm{X}$ ou $\mathrm{X}+\mathrm{Y}$. Ce sont entre autres, des, certains, beaucoup de, de nombreux, bon nombre de, un grand nombre de, le plus grand nombre de, la majorité de, une partie de, une tranche, etc. qui sont valablement utilisables dans presque tous les énoncés du corpus, en tenant compte, bien sûr, des spécificités de chaque énoncé. Du reste, dans bien d'autres éléments du corpus, les numéraux cardinaux peuvent servir également pour désigner les $\mathrm{X}$ ou $\mathrm{X}+\mathrm{Y}$, notamment lorsque l'on peut donner le chiffre exact, précisément en ce qui concerne les noms comptables. D'autres structures encore, déterminant défini $+X$ ou déterminant $+X+Y$, ont besoin d'être complétées par des satellites ou des expansions pour produire un sens objectif. Dans ce dernier cas, le déterminant défini 
demeure, mais on actualise le nom ou le GN. Cette actualisation les débarrasse de toute surdétermination. C'est par exemple le cas des exemples 20, 21, 26 du corpus.

Pour revenir à la forme schématisée de départ, on pourrait avoir les structures suivantes :

- déterminant indéfini $+\mathrm{Y}$ ou déterminant indéfini $+\mathrm{X}+\mathrm{Y}$

- déterminant indéfini $+\mathrm{X}+\mathrm{Y}+\mathrm{Z}$

- ou déterminant défini $\mathrm{X}+\mathrm{Y}+\mathrm{Z}$

$\mathrm{Z}$ constitue une expansion mobile (car pouvant se placer avant ou après $\mathrm{X}$ ou Y) qui lève toute ambiguïté qui tient à l'usage du déterminant défini pluriel et permet d'éclairer davantage le sens de l'énoncé.

A ce stade de notre analyse, il importe de jeter un regard sur les auteurs des emplois excessifs.

\section{II.2. Les auteurs des emplois abusifs}

Il peut sembler paradoxal de convoquer et d'aborder la question des auteurs dans notre analyse. Pour donc lever ce paradoxe, signalons que «la subjectivité langagière » est omniprésente et, de même «qu'il est bien peu de mots qui réchappent du naufrage de l'objectivité » (Kerbrat 147), il est aussi peu d'énoncés, dans la presse écrite notamment, qui n'aient pas de relents de subjectivité. Ainsi, la prise en compte de la thématique développée (politique, faits de société, etc.), de la situation sociopolitique du pays (sortant d'une crise aigüe) voire même des journaux (dont la plupart sont généralement très proches de partis politiques) conduit même à cette conclusion. C'est pourquoi il importe de connaitre les différents auteurs des divers emplois, pour pouvoir déceler leurs intentions.

Le corpus (titres et exploitation de titres) laisse clairement apparaitre plusieurs niveaux de responsabilité quant aux auteurs des énoncés, dont aux moins deux. Il s'agit d'abord des instances de rédaction. En d'autres termes, ce sont les responsables impliqués dans la rédaction du journal. Il s'agit également de ceux dont les énoncés sont rapportés directement. Somme toute, les auteurs putatifs des énoncés sont les «différents niveaux d'énonciation » (22), «la chaine d'émetteurs [...] tant réels que fictifs » (Jakobson 206), y compris ceux qui jouent un simple rôle de relais et qui se contentent de citer l'émetteur originel.

Pour les titres, les auteurs sont d'office les instances de rédaction du journal, lorsqu'aucune trace d'un émetteur, autre que celles des niveaux d'énonciation du journal, n'apparait. Cela ne signifie guère que d'autres émetteurs n'interviennent pas à ce niveau. En effet, si le titre est du discours rapporté directement ou s'il épouse les caractéristiques d'une 
des formes hybrides du discours direct, notamment la forme sans guillemets, alors son émetteur pourrait différer des instances d'énonciation. Ici, ce n'est pas le cas puisque le corpus ne présente pas une telle configuration. Aussi, pouvons-nous conclure que les auteurs des titres de journaux dans notre corpus sont les instances de rédaction.

Cependant, des énoncés rapportés figurent dans le corpus, le 2 et le 28. Les marques $\mathrm{du}$ discours direct, les guillemets et les indices de l'énonciation, notamment les pronoms, augurent une telle conclusion. Pour être plus précis, ces propos ont été tenus par maitre Baroan, avocat de l'ex-président Gbagbo (2) et par l'actuel ministre de l'intérieur (28). Ils ont été rapportés directement, pour le 2, et, sans guillemets, pour le 28. Ainsi, même si ces énoncés sont imputables aux instances de rédaction, la distance que ces dernières prennent les dédouane. Néanmoins, des cas complexes se rencontrent.

Compte tenu de la complexité des émetteurs, il arrive que l'identification des auteurs ne soit pas aussi aisée que dans les deux précédents cas. Car, en l'absence de traces de discours rapporté et d'indices en présence desquels on repère l'émetteur, l'interprétation reste ouverte. Les deux niveaux de responsabilité précédemment évoqués peuvent, et à juste titre, héberger ces énoncés. Cependant qu'il est difficile d'en trouver exactement l'auteur, à moins de rencontrer le rédacteur de l'article ou d'avoir été témoin des faits ayant suscité une telle énonciation, ces énoncés ont un auteur putatif ou de fait : le journaliste.

Au total, l'auteur des usages impropres du déterminant défini pluriel est complexe. Les instances d'énonciation du journal sont, dans tous les cas, même à un degré moindre, en première ligne. Elles en sont les auteurs, c'est le moins qu'on puisse dire. Au demeurant, tout autre auteur de discours, dont tout ou partie se retrouve dans la presse, peut du reste être auteur. In fine, la présomption d'usage abusif du déterminant défini pluriel pèse sur tous les membres de la chaine des émetteurs de l'écrit de presse, avec une certitude quasi élevée pour les instances de presse. Or, puisqu'il est difficile pour tout individu de décrire les choses, la nature ou les faits, en général, avec une impartialité absolue, et que les mots de la langue fonctionnent comme des praxèmes, c'est-à-dire qu'ils connotent à des degrés divers les différentes praxis, on peut affirmer que ces usages, à n'en point douter ne sont pas fortuits. Quels sont donc le sens et les valeurs de tels emplois?

\section{Sens et valeurs des emplois abusifs du déterminant défini pluriel}

Il est reconnu au langage, au regard de ses propriétés spécifiques, la possibilité d'utiliser ce système à plusieurs fins (persuader, convaincre...). La pratique langagière n'est plus comme un simple acte, elle se conçoit comme un acte utilitaire. Ainsi, le choix de 
l'article défini pluriel dans des environnements qui appellent des indéfinis tient au style, à certaines fonctions du journal et à une sorte de défense ou de protection de faces.

\section{III.1.Une question de style : l'hyperbole et la concision}

L'usage tendancieux de l'article défini pluriel relève d'abord de l'hyperbole. En effet, les journalistes, dans leurs écrits, font fréquemment usage de figures de style. Et, parmi celles qui sont sollicitées, figure l'hyperbole. Elle procède de la transformation sémantique par exagération ou amplification du sens d'une réalité. L'usage abusif des déterminants définis fonctionne justement comme ce procédé, car l'émetteur s'en sert pour jouer sur l'intensité des $\mathrm{X}$ ou des $\mathrm{X}+\mathrm{Y}$ dans le sens de l'accroissement. Les énoncés foisonnent notre corpus qui mettent en relief des détails. Suivant notre raisonnement précédent, on comprend qu'il y a bel et bien de l'exagération dans de nombreux énoncés du corpus. Ce sont les énoncés 1, 3, 4, 7, $8,11,12,17,18 \ldots$ Par exemple, écrire que les $S G$ soutiennent tous Bédié n'est pas exact, tout comme écrire que les barons sont en colère contre lui. C'est exprimer au-delà de la vérité. Il faut nuancer les propos. Les structures appropriées étant relevées et expliquées, nous n’y reviendrons plus.

Par ailleurs, le souci de concision est aussi l'une des raisons justificatives des emplois. En effet, le titre doit être concis et condenser toute l'information. Or tout ce qui est résumé perd nécessairement une partie. La règle ici, c'est de ne pas perdre et mieux, de surenchérir. Du point de vue syntaxique, les structures suivantes : déterminant défini $+X$ ou déterminant $+X+Y$ sont plus brèves que celles-ci : déterminant indéfini $+X+Y+Z$ ou déterminant défini $X+Y+Z$.

De plus, même s'ils peuvent paraitre mineurs, le souci de l'économie de l'espace et l'absence de rigueur dans l'utilisation des déterminants guident les choix des journalistes.

\section{III.2. Fonctions d'accroche, d'incitation et d'information du journal}

Le journal est avant tout un produit. Il est destiné à la vente. Et, comme tel, il doit attirer le lecteur et l'inciter à l'achat. L'art de la grandiloquence est, en la matière, une stratégie qui vaut son pesant d'or. Il faut « capter l'intérêt du lecteur » (Helmore 5), susciter obrepticement, chez lui, l'envie de lire le contenu. Ce qui le pousse à acheter. De plus, la première fonction du journal est l'information, c'est-à-dire, d'abord la transmission des nouvelles. Cette fonction, qui vise à la fois à faire connaitre des faits et exprimer des opinions, à exposer et à expliquer, a évidemment des implications, puisqu'elle doit aussi coïncider avec les attentes du lecteur pour modifier son comportement. Par la suite, il faut veiller à fidéliser le lecteur en lui servant les informations dont il a besoin. 


\section{III.3. La défense de faces}

C'est la rhétorique que nous interrogeons, puisque l'écrit de presse lui emprunte les trois pôles sur lesquels elle agit, c'est-à-dire le logos, l'éthos et le pathos. La quête de ces trois intentions se présente comme une sorte de défense de faces. Le dit ou l'écrit de presse, en effet, doit donner la preuve de la véracité de ce qui est dit ou écrit. En témoignent les énoncés (2) et (28) non-inscrits au compte du journal. Le journal doit par ailleurs concilier la bienveillance du lectorat et éveiller en lui toutes les émotions qui sont utiles à la cause défendue ou à défendre. En la matière, les expressions fédératrice aux Ivoiriens, ou classificatoire les pro-Gbagbo ou les pro-Ouattara qui, en réalité, désignent partie, remplissent fort bien ce rôle.

Le style que prend le journal capte d'abord l'attention du lectorat. Une fois que cette mission est accomplie, le journal a désormais la confiance qui le crédibilise aux yeux du lectorat. Il joue aussi sur la sensibilité de ce dernier. A travers les emplois excessifs de l'article défini les, on assiste à une projection du débat politique. En effet, qu'il s'agisse de journal ou d'intervenants proches du pouvoir, de l'opposition, d'un parti politique ou de personnes influentes, l'explosion sémantique a une connotation positive ou négative. On se défend et on enfonce l'autre. Ainsi, le Mandat, journal proche du PDCI et de son mentor, ne peut que relayer 11. De même, Notre Voie, proche du FPI ne peut que constater les énoncés 29 ([tous les...] pour déplorer), 20 et 21 (pour montrer l'incapacité des dirigeants actuels). Aussi, 28 (propos du ministre de l'intérieur) et 13 prospèrent dans le Patriote et l'Expression qui sont proches des dirigeants et du RDR.

\section{Conclusion}

En définitive, l'usage de l'article défini pluriel trahit le consensus minimum sur lequel repose l'emploi des articles. Cela a pour conséquence d'exagérer ou d'amplifier le sens des mots qu'il détermine, à charge ou à décharge des destinataires des énoncés. Au demeurant, cet emploi inexact, à plusieurs niveaux de responsabilité, est déterminé par plusieurs facteurs qui s'organisent en des chaînes signifiantes différentes, chacune d'elles possédant sa cohérence et requérant une interprétation particulière. Cet usage participe de la construction du sens en contexte. 


\section{Corpus}

L'expression 1164

L'inter 4416

Le jour 2798

Le mandat 1154

Nord sud 2406

Notre voie 4464, 4472, 4483

Le nouveau courrier 732

Le patriote 3975, 4108

Le quotidien d'Abidjan 0914

\section{Bibliographie}

Amossy, R. L'argumentation dans le discours. Discours politique, littérature d'idées, fiction. Paris : Colin, 2006.

Arrivé M., F. Gadet et M. Galmiche. La grammaire d'aujourd'hui : guide alphabétique de linguistique française. Paris : Flammarion, 1986.

Helmore, K. A.B.C. de la presse écrite, Paris : Nouveaux Horizons, 1995.

Jakobson, Roman. Question de poétique. Paris : Seuil, 1973.

Kerbrat-Orecchioni, C. L'énonciation de la subjectivité dans le langage. Paris : Colin, 1980.

Koren, R. Les enjeux éthiques de l'écriture de presse et la mise en mots du terrorisme. Paris : Harmattan, 1996. 